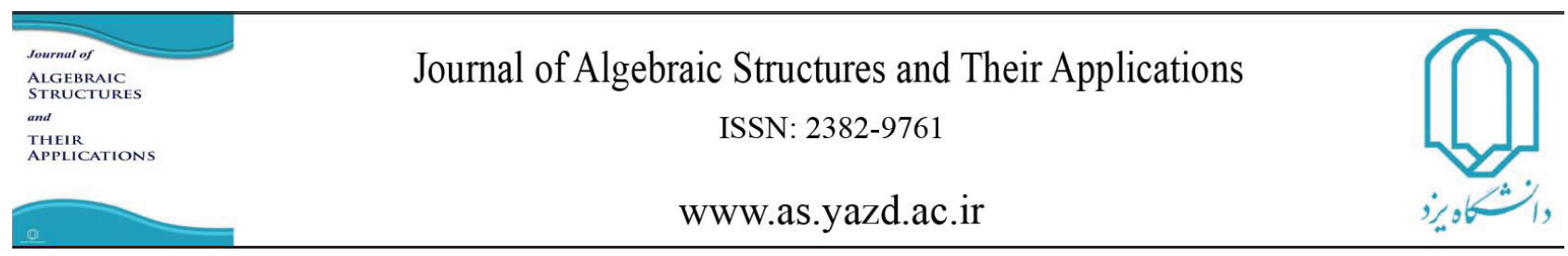

Algebraic Structures and Their Applications Vol. 6 No. 1 ( 2019 ) pp 103-114.

\title{
HYPERRINGS WHICH EVERY ELEMENT IS SUM OF AN IDEMPOTENT AND NILPOTENT
}

\author{
YAHYA TALEBI* AND MARZIYEH FARZINEJAD
}

\begin{abstract}
In this paper, we define a generalized of clean of Krasner hyperrings for general hyperrings based on the notation of nilpotent elements of a general hyperring $R$, named nil clean hyperring. We examine characterization of this kind of hyperrings and finally, we obtain some relations of nil clean general hyperrings with other hyperrings.
\end{abstract}

\section{INTRODUCTION}

First time, the hyperstructure theory was introduced by Marty in [1.3]. Later many applications of this theory were obtained by some researchers (see $[\underline{6}, \mathbf{7}, 18]$ ).

Marty defined the notion of the hypergroups. Then several researchers have been endowed it, (for example see Mittas in [14]).

The theory of hyperstructure has been widely reviewed $[\mathbf{6}, \mathbf{7}, \mathbf{8}]$.

The hyperrings were introduced by Krasner in [1T]. A Krasner hyperring is a nonempty set $R$ with a hyperoperation (the addition) and binary operation (the multiplication) such that $(R,+)$ is a cononical hypergroup, $(R, \cdot)$ is a semigroup and the multiplication is distributive

DOI : $10.29252 /$ as. 2019.1360

MSC(2010): Primary 16Y99; Secondary 20N20

Keywords: Hyperring, General hyperring, Nil clean general hyperring.

Received: 10 April 2018, Accepted: 20 April 2019.

*Corresponding author

(C) 2019 Yazd University. 
with respect to the addition. The hyperrings were studied by Nakassis [15], Massouros [I2], and by Ameri and Norouzi [1, [2] and others.

Also, there exists the several types of hyperrings that was widely discussed in $[8]$. Following [8] a general hyperring is a general type of hyperrings that both of the addition and multiplication are hyperoperations.

Clean rings were difined as a subclass of exchange rings by Nicholson [16]. Following [16], an elemnt $x$ of a ring $R$ is said to be clean provided that there exists an idempotent $e \in R$ and a unit $u \in R$ such that $x=e+u$, and $R$ is clean if every element is clean. Later, many authors have have studied clean rings and generalized them (see [ [5, 9] ).

Following $[\overline{9}]$, Alexande J. Diesl difined Nil clean rings as a generalization of clean rings. He called an element $a$ of a ring $R$ is nil clean if it can be written as a sum of an idempotent and a nilpotent, and $R$ is nil clean ring if every element has such decomposition. In this paper, based on this definition of nil clean rings $([9])$, we define nil clean hyperring and we study various properties of such rings.

\section{Preliminaries}

In this section, we recall some definitions concerning hyperrings theory that we used in this paper.

A canonical hypergroup [14] is a nonempty set $H$ with an additive hyperoperation + : $H \times H \rightarrow p^{*}(H)$ satisfying the following properties:

i) For any $a, b, c \in H, a+(b+c)=(a+b)+c$;

ii) For any $a, b \in H, a+b=b+a$;

iii) There exists $0 \in H$ such that $0+a=a+0=\{a\}$ for all $a \in R$;

iv) For every $a \in H$, there exists a unique element $a^{\prime} \in H$, such that $0 \in a+a^{\prime}$ (we write $-a$ instead of $a^{\prime}$ and we call it the opposite of $a$ ).

v) $c \in a+b$ implies that $b \in-a+c$ and $a \in c-b$.

Definition 2.1. A multi valued system $(R,+, \cdot)$ is said to be

(1) general hyperring, if

$\left.a_{1}\right)(R,+)$ is a hypergroup;

$\left.a_{2}\right)(R, \cdot)$ is a semihypergroup;

$\left.a_{3}\right)$ "." is distributive with respect to "+",

(2) Krasner hyperring, if

$\left.b_{1}\right)(R,+)$ is a canonical hypergroup;

$\left.b_{2}\right)(R, \cdot)$ is a semigroup having zero as a bilaterally absorbing, i.e, for all $a \in R$ we have $0 \cdot a=a \cdot 0=0$;

$\left.b_{3}\right) a \cdot(b+c)=a \cdot b+a \cdot c$ and $(b+c) \cdot a=b \cdot a+c \cdot a$, for all $a, b, c \in R$. 
(3) Multiplicative hyperring, if

$\left.c_{1}\right)(R,+)$ is a commutative group;

$\left.c_{2}\right)(R, \cdot)$ is a semihypergroup;

$\left.c_{3}\right)$ for every $a, b, c \in R$ we have,

i) $a \cdot(b+c) \subseteq a \cdot b+a \cdot c$ and $(b+c) \cdot a \subseteq b \cdot a+c \cdot a$

ii) $a \cdot(-b)=(-a) \cdot b=-(a \cdot b)$.

In this paper we use of general hyperring.

A nonempty subset $I$ of a hyperring $R$ is a hyperideal, if $a, b \in I$ implies $a-b \subseteq I$ and for $r \in R, r \cdot a \cup a \cdot r \subseteq I$. It is called a normal hyperideal when $x+I-x \subseteq I$ for all $x \in R$.

A proper hyperideal $M$ of $R$ is called a maxial hyperideal, if the only hyperideals of $R$ that contain $M$ are $M$ itself and $R$.

A proper hyperideal $P$ of a hyperring $R$ is called a prime hyperideal of $R$ if $a \cdot b \subseteq P$ implies that $a \in P$ or $b \in P$, for all $a, b \in R$.

It is well known that, in a commutative hyperring $R$ with a unit element, there exists a maximal hyperideal of $R$ containing $I$, for every proper hyperideal $I$ of $R$.

Recall, a nonzero hyperring $R$ having a unique maximal hyperideal is called a local hyperring.

The intersection of all maximal hyperideals of $R$ is called the Jacobson radical of $R$ and denoted by $J(R)$.

Moreover, for $A, B \subseteq R$ and $x \in R$,

$$
\begin{gathered}
A+B=\cup\{a+b \mid a \in A, b \in B\} \\
A B=\cup\{a b \mid a \in A, b \in B\},
\end{gathered}
$$

and $A+x=A+\{x\}, x+B=\{x\}+B$ and also $-A=\{-a \mid a \in A\}$.

The following elementary facts in a hyperring follows from the axiom: $-(-x)=x$ and $-(x+y)=-x-y$. Also, for each $a, b, c, d \in R$, we have

$$
(a+b) \cdot(c+d) \subseteq a \cdot c+b \cdot c+a \cdot d+b \cdot d,
$$

whenever $R$ is commutative

$$
\begin{gathered}
(a+b) \cdot(c+d)=a \cdot c+b \cdot c+a \cdot d+b \cdot d, \\
-(a b)=(-a) b=a(-b) .
\end{gathered}
$$

Definition 2.2. Let $R_{1}$ and $R_{2}$ be hyperrings. A mapping $f$ from $R_{1}$ into $R_{2}$ is said to be 
i) a homomorphism if for all $a, b \in R_{1}$

$$
\begin{gathered}
f(a+b) \subseteq f(a)+f(b), \\
f(a b)=f(a) \cdot f(b)
\end{gathered}
$$

ii) good (or strong) homomorphism if in (i) equality holds.

iii) epimorphism if $f$ is a surjective homomorphism and also if for every $a_{2}, b_{2} \in R_{2}$ the following holds:

$$
\left(\forall y \in a_{2}+b_{2}\right)\left(\exists a_{1}, b_{1} \in R\right)\left(\exists x \in a_{1}+b_{1}\right), f\left(a_{1}\right)=a_{2}, f\left(b_{1}\right)=b_{2}, f(x)=y
$$

iv) isomorphism if it is a bijective good homomorphism.

\section{MAin RESUlts}

Following [4], Amozegar and Talebi defined the notation of clean for Krasner hyperrings, and investigated some properties such rings. Now, we generalize this definition with nilpotent element for general hyperring.

In this paper, by a hyperring $R$, we mean a general hyperring which is satisfied in the following conditions:

(1) there exists $0 \in R$, such that $0+x=\{x\}$ and $x \cdot 0=0 \cdot x=\{0\}$ for all $x \in R$;

(2) for every $x \in R$ there exists a unique $x^{\prime} \in R$ such that $0 \in x+x^{\prime}$ (we write $-x$ instead of $x^{\prime}$ and we call it the opposite of $x$ );

(3) $z \in x+y$ implies that $y \in-x+z$ and $x \in z-y$, that is $(R,+)$ is revesible;

(4) $R$ is commutative with respect to both hyperoperations + and $\cdot$;

(5) $R$ with the identity element 1 , i.e, for every $x \in R, 1 \cdot x=x \cdot 1=\{x\}$.

Definition 3.1. Let $(R,+, \cdot)$ be a hyperring. $x \in R$ is said to be

(1) idempotent, if $\{x\}=x \cdot x=x^{2}$;

(2) weak idempotent, if $x \in x^{2}$.

Definition 3.2. The element $x$ of $(R,+, \cdot)$ is called

(1) nilpotent, if $\{0\}=x^{n}$ for some $n>0$;

(2) weak nilpotent, if $0 \in x^{n}$ for some $n>0$.

A hyperideal $I$ of $R$ is a (weak) nil hyperideal if each element of $I$ is a (weak) nilpotent element.

A nonzero hyperideal $I$ of hyperring $R$ is nil normal hyperideal, if it is normal hyperideal and nil.

Definition 3.3. Let $(R,+, \cdot)$ be a hyperring. The element $x \in R$ is said to be 
(1) unite, if there exists $y \in R$ such that $\{1\}=x \cdot y$;

(2) weak unit, if $1 \in x \cdot y$ for $y \in R$.

We denote the set of idempotent (weak idempotent), unit (weak unit) and nilpotent (weak nilpotent) elements of $R$ by $\operatorname{Id}(R)\left(\operatorname{Id}_{w}(R)\right), U(R)\left(U_{w}(R)\right), N(R)\left(N_{w}(R)\right)$, respectively.

Lemma 3.4. Let $R$ be a hyperring and $r \in R$. Then $r \in J(R)$ if and only if $1-r x \subseteq U(R)$ for every $x \in R$.

Proof. Suppose that $r \in J(R)$ and $1-r x \nsubseteq U(R)$, for some $x \in R$. Let $a \in 1-r x \nsubseteq U(R)$, then there exists a maximal hyperideal $M$ of $R$ such that $a \in M$. On the other hand, $r \in M$, then $1 \in a+r x \subseteq a+M \subseteq M$, a contradiction.

Conversely, suppose $1-r x \subseteq U(R)$ for every $x \in R$. Let $M$ be a maximal hyperideal of $R$, we show that $r \in M$. Suppose $r \notin M$, then there exist $m \in M$ and $a \in R$ such that $1 \in r a+m$ by the maximality $M$. So $m \in 1-r a \subseteq U(R)$ a contradiction. Then for each maximal hyperideal $M$ of $R, r \in M$ and so $r \in J(R)$.

Definition 3.5. Let $R$ be a hyperring. An element $x \in R$ is (weak) nil clean element if the element $x$ has a representation in the form $x \in e+n$ where $e \in \operatorname{Id}(R)\left(\operatorname{Id}_{w}(R)\right)$ and $n \in N(R)\left(N_{w}(R)\right)$. Also, hyperring $R$ is (weak) nil clean hyperring if every element has such representation.

Example 3.6. Let $R=\{0, a, b, c\}$ be a set with hyperoperation + and $\cdot$, defined as follows:

\begin{tabular}{|c|c|c|c|c|c|c|c|c|c|}
\hline+ & 0 & $a$ & $b$ & $c$ & . & 0 & $a$ & $b$ & $c$ \\
\hline 0 & $\{0\}$ & $\{a\}$ & $\{b\}$ & $\{c\}$ & 0 & $\{0\}$ & $\{0\}$ & $\{0\}$ & $\{0\}$ \\
\hline$a$ & $\{a\}$ & $\{0\}$ & $\{c\}$ & $\{b\}$ & $a$ & $\{0\}$ & $\{a\}$ & $\{b\}$ & $\{c\}$ \\
\hline$b$ & $\{b\}$ & $\{c\}$ & $\{0\}$ & $\{a\}$ & $b$ & $\{0\}$ & $\{a\}$ & $\{b\}$ & $\{c\}$ \\
\hline$c$ & $\{c\}$ & $\{b\}$ & $\{a\}$ & $\{0\}$ & $c$ & $\{0\}$ & $\{0\}$ & $\{0\}$ & $\{0\}$ \\
\hline
\end{tabular}

Then $(R, \oplus, \odot)$ is a nil clean hyperring.

Since every nilpotent (idempotent) element is also weak nilpotent (weak idempotent), then every nil clean hyperring is a weak nil clean hyperring. But there exists weak nil clean hyperring that is not nil clean hyprring.

Example 3.7. Consider $R=\{0,1\}$ with the following hyper operations:

\begin{tabular}{|c|c|c|c|c|}
\hline+ & 0 & . & 0 & 1 \\
\hline 0 & $\{0\} \quad\{1\}$ & 0 & $\{0\}$ & $\{0\}$ \\
\hline 1 & $\{1\} \quad R$ & 1 & $\{0\}$ & $R$ \\
\hline
\end{tabular}


It is easy to verify that $(R, \oplus, \odot)$ is a weak nil clean hyperring but is not nil clean hyperring.

Definition 3.8. Let $R$ be hyperring. The element $a \in R$ is (weak) unipotent if there exists (weak) nilpotent element $b \in R$ such that $a \in 1+b$.

Corollary 3.9. Each (weak) unipotent element in a hyperring $R$ is a (weak) nil clean element.

Let $I$ be a hyperideal of the hyperring $(R,+, \cdot)$. The set $\frac{R}{I}=\{x+I \mid x \in R\}$ with respect to the following hyperoperations

$$
\begin{aligned}
& (x+I) \oplus(y+I)=\{z+I \mid z \in x+y\}, \\
& (x+I) \odot(y+I)=\{t+I \mid t \in x \cdot y\}
\end{aligned}
$$

for all $x, y \in R$, form the factor hyperring.

We use $\bar{x}$ to stand for $x+I \in \frac{R}{I}$, for $x \in R$ and hyperideal $I$ of $R$.

Definition 3.10. Let $\left(\frac{R}{I},+, \cdot\right)$ be a hyperring and $x \in R . \bar{x} \in \frac{R}{I}$ is said to be

(1) idempotent, if $\{\bar{x}\}=\bar{x} \cdot \bar{x}=\bar{x}^{2}$, and weak idempotent, if $\bar{x} \in \bar{x}^{2}$;

(2) nilpotent, if $\{\overline{0}\}=\bar{x}^{n}$, and weak nilpotent, if $\overline{0} \in \bar{x}^{n}$, for some $n>0$;

(3) unit, if there exists $y \in R$ such that $\{\overline{1}\}=\bar{x} \cdot \bar{y}$, and weak unit, if $\overline{1} \in \bar{x} \cdot \bar{y}$ for $y \in R$.

Proposition 3.11. Let $I$ be a hyperideal of hyperring $R$. If $R$ is (weak) nil clean hyperring then $\frac{R}{I}$ is weak nil clean hyperring.

Proof. Let $x+I \in \frac{R}{I}$, where $x \in R$. Then $x \in e+b$, where $\{e\}=e^{2}$ and $\{0\}=b^{n}$ for some $n>0$, by hypothesis. Therefore, $x+I \in(e+I) \oplus(b+I)$, we show that $e+I \in \operatorname{Id}\left(\frac{R}{I}\right)$ and $b+I \in N_{w}\left(\frac{R}{I}\right)$. We have

$$
(e+I)^{2}=(e+I) \odot(e+I)=\left\{\ell+I \mid \ell \in e \cdot e=e^{2}=\{e\}\right\}=\{e+I\} .
$$

Then $e+I \in \operatorname{Id}\left(\frac{R}{I}\right)$. On the other hand

$$
\begin{aligned}
(b+I)^{n}=(b+I) \odot(b+I)^{n-1} & =\bigcup\left\{\left\{k+I \mid k \in_{t \in b^{n-1}} b \cdot t \subseteq b^{n}=\{0\}\right\}\right\} \\
& =\bigcup\{\{0+I\}\}=\{\{I\}\},
\end{aligned}
$$

then $b+I \in N_{w}\left(\frac{R}{I}\right)$. Then the results follows.

Proposition 3.12. Let $R$ be a hyperring and I a nil normal hyperideal of hyperring $R$. If $\frac{R}{I}$ is a (weak) nil clean hyperring, then $R$ is a weak nil clean hyperring. 
Proof. Suppose $x \in R$ and $\bar{x} \in \bar{e}+\bar{n}$ where $\{\bar{e}\}=\bar{e}^{2}=\bar{e} \cdot \bar{e}$ and $\{\overline{0}\}=\bar{n}^{k}$ for some $k>0$. By hyporthesis, we lift $\bar{e}$ to an idempotent $e \in R$. We have $\bar{x} \in \bar{e}+\bar{n}=\{t+I \mid t \in e+n\}$, then there exists $t \in e+n$ such that $x+I=t+I$. Since $I$ is normal hyperideal, we have

$$
x-e+I=t-e+I \subseteq e+n-e+I \subseteq n+I .
$$

Therefore,

$$
x-e+I=\cup\{c+I \mid c \in x-e\} \subseteq N\left(\frac{R}{I}\right) .
$$

Then $c+I$ is a nilpotent element in $\frac{R}{I}$, so there exists $n \in \mathbb{N}$ such that $\{\overline{0}\}=\bar{c}^{n}$, then

$$
I \subseteq(c+I)^{n}=(c+I) \cdot(c+I)^{n-1} \subseteq \cup\left\{b+I \mid b \in c^{n}\right\}
$$

So, there exists $a \in c^{n}$ and $i \in I$ such that $i \in a+I$. Then $a \in i-I \subseteq I$, therefore $a \in I$ is a nilpotent element in $R$. So $\{0\}=a^{\ell} \in\left(c^{n}\right)^{\ell}$ for some $\ell>0$, which implies $c$ is a weak nilpotent element in $R$. On the other hand, $c \in x-e$, then $x \in e+c$. Hence $R$ is a weak nil clean hyperring.

Any finite direct product of nil clean hyperring is nil clean hyperring. But there exists an infinite product of nil clean hyperrings that is not.

Example 3.13. Consider the hyperring $R=\mathbb{Z}_{2} \times \mathbb{Z}_{4} \times \mathbb{Z}_{8} \ldots$ which the rings $\mathbb{Z}_{n}$ with hyperoperations $\oplus$ and $\odot$ that $\bar{a} \oplus \bar{b}$ and $\bar{a} \cdot \bar{b}$ are the remainder of $\frac{a+b}{n}$ and $\frac{a \cdot b}{n}$, respectively, for all $\bar{a}, \bar{b} \in \mathbb{Z}_{n}$, are nil clean hyperrings, for $n=2,4,8,16, \ldots$. But the ring $R$ is not nil clean hyperring. Just consider the element $r=(0,2,2,2, \ldots) \in R$.

Recall a hyperring $R$ is (weak) Boolean when each element is an (weak) idempotent in $R$, and hyperring $R$ is reduced if it does not have any non-zero nilpotent element.

Corollary 3.14. Each (weak) Boolean hyperring is (weak) nil clean hyperring.

Corollary 3.15. Let $R$ is a reduced hyperring, then $R$ is nil clean hyperring if and only if $R$ is Boolean hyperring.

Proof. Let $a \in R$, then $a \in e+b$ for some $e \in \operatorname{Id}(R)$ and $b \in \operatorname{Nil}(R)$. Since $R$ is reduced, then $b=0$ and $a \in e+0=\{e\}$, therefore $a=e$ and the result follows, the converse is clear.

Proposition 3.16. If $b$ is a (weak) nilpotent element in a hyperring $R$, then the set $1 \pm b$ is a weakly unit set [2]. 
Recall a hyperring $R$ is (weak) clean hyperring if for every element $x \in R$ there exists $u \in U(R)\left(U_{w}(R)\right)$ and $e \in \operatorname{Id}(R)\left(\operatorname{Id}_{w}(R)\right)$ such that $x \in e+u$.

Proposition 3.17. If $R$ is a (weak) nil clean hyperring, then $R$ is a weak clean hyperring.

Proof. Let $r \in R$ and $a \in r-1 \subseteq R$. Therefore $r \in a+1$, so there exist $e \in \operatorname{Id}(R)$ and $b \in N(R)$ such that $a \in e+b$ by hyporthesis. Thus we have

$$
r \in a+1 \subseteq(e+b)+1=e+(b+1)=\cup\{e+x \mid x \in b+1\} .
$$

Therefore, there exists $c \in b+1$ such that $r \in e+c$. Hence the result follows by Proposition 3.16

Example 3.18. There exists a weak clean hyperring that is not nil clean hyperring. Let $R=\{0,1,2\}$ with the hyperoperation $\oplus$ and $\odot$, the following,

\begin{tabular}{c|ccccc|ccc}
$\oplus$ & 0 & 1 & 2 & & $\odot$ & 0 & 1 & 2 \\
\hline 0 & $\{0\}$ & $\{1\}$ & $\{2\}$ & & 0 & $\{0\}$ & $\{0\}$ & $\{0\}$ \\
1 & $\{1\}$ & $\{1\}$ & $R$ & 1 & $\{0\}$ & $\{1\}$ & $\{2\}$ \\
2 & $\{2\}$ & $R$ & $\{2\}$ & & $\{0\}$ & $\{2\}$ & $\{1\}$
\end{tabular}

$R$ is a clean hyperring that is not nil clean hyperring. Because of the element 2 in $R$ does not have any representation as nil clean element in $R$.

Proposition 3.19. If $R$ is a hyperring, then every (weak) nilpotent element is contained in $J(R)$.

Proof. Let $b \in R$ is a nilpotent element with the nilpotency index $n$. We have $\{0\}=a^{n} \cdot\{0\}=$ $a^{n} \cdot b^{n}=(a b)^{n}$ for every $a \in R$. So for all $\ell \in a \cdot b$ we have $\{0\}=\ell^{n}$ ans thus $1-\ell \subseteq U_{w}(R)$ by Proposition B.T6.

Proposition 3.20. If $R$ is a (weak) nil clean hyperring, then $J(R)$ is (weak) nil hyperideal of $R$.

Proof. Let $a \in J(R)$, then $a \in e+b$ where $\{e\}=e^{2}$ and $\{0\}=b^{n}$. Thus $b \in a-e$ and we have

$$
\{0\}=b^{n} \subseteq(a-e)^{n} \subseteq \sum_{0}^{n-1}\left(\begin{array}{l}
n \\
k
\end{array}\right) a^{n-k} e^{k}+e^{n}
$$

by bionomial Theorem. Set $A=\sum_{0}^{n-1}\left(\begin{array}{l}n \\ k\end{array}\right) a^{n-k} e^{k}$, since $a \in J(R)$, then $A \subseteq J(R)$. Thus

$$
0 \in A+e^{n} \subseteq J+e^{n} \subseteq J+\{\{e\}\} .
$$

Therefore, there exists $t \in J$ such that $0 \in t+e$, then $e \in 0-t \subseteq J(R)$. So $e=0$ and then $a \in J(R)$ is a nilpotent element. Hence the result follows. 
Alg. Struc. Appl. Vol. 6 No. 1 (2019) 103-114.

Proposition 3.21. Let $R$ is a hyperring. Then the following conditions are hold:

(1) If $R$ is a nil clean hyperring with $J(R)=0$, then $R$ is reduced and therefore Boolean hyperring;

2) If $J(R)$ is normal hyperideal, then $R$ is a weak nil clean hyperring if and only if $\frac{R}{J}$ is weak Boolean hyperring and $J(R)$ is weak nil hyperideal.

Proof. (1) It is straightforward. (2) Let $a \in J(R)$, then $a \in e+b$ where $e \in e^{2}$ and $0 \in b^{n}$ for some $n>0$. Thus $e \in a-b \subseteq J(R)$ by Proposition B.I.9. Therefore, $e=0$ and $J(R)$ is weak nil hyperideal. On the other hand, $\frac{R}{J}$ is weak nil clean hyperring by Proposition $\mathbf{B} . \amalg$, and also $J\left(\frac{R}{J}\right)=0$, then the result follows by (1).

Conversely, suppose $x \in R$, then $x+J \in \frac{R}{J}$ is an weak idempotent. Since $J(R)$ is weak nil hyperideal, then there exists an idempotent $e$ in $R$ such that $x+J=e+J$, then $x \in e+J$ and since $J(R)$ is normal hyperideal, we have $x-e \subseteq e+J-e \subseteq J$. Therefore, we have

$$
x \in x+0 \subseteq(x-e)+e=\cup\{a+e \mid a \in x-e\} .
$$

Then there exists $r \in x-e$ is weak nilpotent and so $x \in r+e$. Hence $R$ is weak nil clean hyperring.

Lemma 3.22. If $R$ is a hyperring, then the following are equivalent:

(1) $R$ has a unique maximal hyperideal;

(2) the set of non-invertible elements of $R$ is closed under addition;

(3) $x \in U(R)\left(U_{w}(R)\right)$ or $(1-x) \subseteq U(R)\left(U_{w}(R)\right)$ for any $x \in R$;

(4) $J(R)=\{x \in R \mid x$ is not invertible $\}$;

(5) $\frac{R}{J(R)}$ is a division hyperring.

Theorem 3.23. Let $R$ is a hyperring with trivial idempotent, then the following are equivalent:

(1) $R$ is nil clean hyperring;

(2) $R$ is local hyperring with $J(R)$ is nil hyperideal and $\frac{R}{J(R)}$ is division hyperring.

Proof. (1) $\Rightarrow(2) . J(R)$ is nil hyperideal by Proposition 3.20. Let $r \in R$ with $r \in e+b$ where $\{e\}=e^{2}$ and $\{0\}=b^{n}$ for some $n>0$. If $e=0$, then $r \in 0+b=\{b\}$ and hence $r$ is nilpotent and $1-r \subseteq U_{w}(R)$ by Proposition [3.16. On the other hand, if $e=1$, then $r \in 1+b \subseteq U_{w}(R)$ by Proposition [.16. Then $R$ is local hyperring.

$(2) \Rightarrow(1)$. Let $r \in R$ and $r \notin U(R)$, then $r \in J(R)$ and $r \in 0+r$. Now, if $r \in U(R)$, then $1-r \subseteq J(R)$, so we have $r \in r+0 \subseteq(r-1)+1=\cup\{x+1 \mid x \in r-1\}$. Therefore, there exists $a \in r-1$ such that $r \in a+1$ and since $J(R)$ is nil hyperideal, then $R$ is nil clean hyperring. 
Proposition 3.24. Let $R$ is a hyperring. The following conditions are equivalent:

(1) $R$ is nil clean hyperring;

(2) for every element $x \in R, x \in b-e$ where $b \in N(R)$ and $e \in \operatorname{Id}(R)$;

(3) for every element $x \in R, x \in b+e$ where $b \in N(R) \cup\{0\}$ and $e \in \operatorname{Id}(R)$;

(4) for every element $x \in R, x \in b-e$ where $b \in N(R) \cup\{0\}$ and $e \in \operatorname{Id}(R)$.

Proof. (1) $\Rightarrow(2)$. Let $x \in R$, then $-x \in R$ and $-x \in b+e$ where $e \in \operatorname{Id}(R)$ and $b \in N(R)$ by hypothesis. We have $x \in-b-e$ where $-b \in N(R)$ and $e \in \operatorname{Id}(R)$. (2) $\Rightarrow(3)$ and (3) $\Rightarrow(4)$ are similar to $(1) \Rightarrow(2)$. (4) $\Rightarrow(1)$. Let $x \in R$. Then $-x \in b-e$ for some $b \in N(R) \cup\{0\}$ and $e \in \operatorname{Id}(R)$. Therefore, $x \in(-b)+e$ where $-b \in N(R)$ and $e \in \operatorname{Id}(R)$. The case $b=0$, then $x \in 0+e$ and the result follows.

Lemma 3.25. If $R$ is a hyperring, then the product of two unit elements is a weakly unit set.

Proof. Let $x$ and $y$ are unit elements with $x \cdot x^{-1}=\{1\}$ and $y \cdot y^{-1}=\{1\}$, respectively. Suppose $t \in x \cdot y$, we have

$$
t \cdot y^{-1} \subseteq(x \cdot y) \cdot y^{-1}=x \cdot\left(y \cdot y^{-1}\right)=x \cdot\{1\}=\{\{x\}\}=0+\{x\} .
$$

Then for every $a \in t \cdot y^{-1}, a \in 0+x$, so

$$
x \in 0-a \subseteq 0-t \cdot y^{-1} \subseteq\{0\}-t \cdot y^{-1}=t \cdot 0-t \cdot y^{-1}=t\left(0-y^{-1}\right) .
$$

Thus we have

$$
1 \in\{1\}=x \cdot x^{-1} \subseteq t\left(0-y^{-1}\right) x^{-1}=t\left(0 \cdot x^{-1}-y^{-1} \cdot x^{-1}\right) .
$$

Hence the result follows.

Proposition 3.26. Let $R$ be a hyperring. Then the sum of an unit and a nilpotent element is a weakly unit set.

Proof. Let $n$ and $u$ be nilpotent element and unite element, respectively, and also $u u^{-1}=\{1\}$. Then $u^{-1} n$ is a nilpotent element. Now we have

$$
u+n \subseteq u+\{1\} \cdot n=u+u u^{-1} n=u\left(1+u^{-1} u\right) \subseteq U_{w}(R)
$$

by Lemma 3.25.

Theorem 3.27. Let $(R,+, \cdot)$ be a multiplicative hyperring. If $R$ is a nil clean hyperring and $I$ is a hyperideal of $R$ such that does not include any non-zero idempotent of $R$, then there exists a maximal hyperideal $K$ of $R$ such that $I \subseteq K$ and does not have any idempotent of $R$. 
Alg. Struc. Appl. Vol. 6 No. 1 (2019) 103-114.

Proof. Let $B=\left\{J \mid J \unlhd R, I \subseteq J\right.$ and $\nexists\{e\}=e^{2} \in R$ s.t. $\left.e \in J\right\}$. Then $\beta \neq \emptyset \cdot(I \in \beta)$. The set $\beta$ is orderd by inclusion, then there exists a hyperideal $L$ of $R$ that is maximal in $\beta$, by using Zorn's Lemma. Let $a, b \in R$ with $a, b \notin L$. Then $L \subseteq L+\langle a\rangle$ and $L \subseteq L+\langle b\rangle$. Since $L$ is maximal hyperideal in $\beta$, then $L+\langle a\rangle$ and $L+<b>\notin \beta$ and so there exist idempotents $e$ and $f$ of $R$ such that $e \in L+\langle a\rangle$ and $f \in L+\langle b\rangle$. This shows $e f \subseteq L+\langle a \cdot b\rangle$.

It follows that $a \cdot b \nsubseteq L$, hence, $L$ is prime. Now we show that $L$ is a maximal hyperideal of $R$. Suppose that $L$ is not maximal, therefore there exists a maximal hyperideal $M$ of $R$ where $L \subsetneq M \subsetneq R$. Choose $a \in M$ while $a \notin L$. Then there exists an idempotent $p$ and a nilpotent element $n$ of $R$ such that $a=p+n$, by hypothesis. Then $n \in L[2]$. On the other hand, $p R(1-p) \subseteq L$. As $L$ is prime, we have $1-p \in L$. Thus $1-a=(1-p)-n \in L \subseteq M$. Then $1-a \in M$, and so $1=(1-a)+a \in M$, a contradiction. Then $L$ is a maximal hyperideal.

Proposition 3.28. Let $R$ be a Krasner hyperring and $a \in R$. the following conditions are hold:

(1) $a \in e+u$, then $\ell(a) \subseteq R\{e\}$ where $e \in \operatorname{Id}(R)$ and $u \in U(R)$.

(2) $a \in e+b$, then $\ell(a) \subseteq R\{1-e\}$ where $e \in \operatorname{Id}(R)$ and $b \in N(R)$.

Proof. (1) Let $r \in \ell(a)$. Then $0=r a \in r(e+u)=r e+r u$. Thus

$$
r u \in 0-r e=r \cdot 0-r e=r(0-e) .
$$

Now, we have

$$
r=r \cdot 1=r \cdot u u^{-1} \in r(0-e) u^{-1}=r u^{-1}(0-e) \subseteq R\{e\} .
$$

(2) Let $r \in \ell(a)$. Then $0=r a \in r(e+b)=r e+r b$. Thus

$$
r b+r \subseteq 0-r e+r=r(0-e+1) .
$$

On the other hand, for every $c \in(b+1)$

$$
r=r \cdot 1 \in r c c^{-1} \subseteq r(b+1) c^{-1} \subseteq r(0-e+1) c^{-1}=r c^{-1}(0+(1-e)) \subseteq R\{1-e\} .
$$

\section{REFERENCES}

[1] R. Ameri, M. Norouzi, New fundamental relation of hyperrings, European J. Combin., 34 (2013) $884-891$.

[2] R. Ameri, M. Norouzi, On commutative hyperring, int. Journal of Algebraic Hyperstructures and its Applications, 1 (2014), No. 1, 45-58.

[3] R. Ameri, M. Norouzi, On multiplication $(m, n)$-hypermodules, European Journal of Combinatorics, 44 (2015), 153-171.

[4] T. Amouzegar, Y. Talebi, On clean hyperrings, Journal of Hyperstructures, 4 (1) (2015), 1-10. 
[5] H. Chen, On strongly J-clean rings, Comm. Algebra 38 (2010), 3790-3804.

[6] P. Corsini, Prolegomena of Hypergroup Theory, Second Eddition Aviani, Editor, (1993).

[7] P. Corsini, V. Leoreanu, Applications of Hyperstructure Theory, Advances in Mathematics. Kluwer Academic Publishers, (2003).

[8] B. Davvaz and V. Leoreanu-Fotea,, Hyperring Theory and Applications, International Academic Press, Palm Harbor, USA, 2007.

[9] A.J. Diesl, Nil clean rings, J. Algebra, 383 (2013), 197-211.

[10] J. Han and W.K. Nicholson, Extensions of clean rings, Comm. Algebra 29 (2001), No. 6, $2589-2595$.

[11] M. Krasner, A Class of Hyperring and Hyperfields, Intern. J. Math. Sci. 6 (1983), 307-312.

[12] C.G. Massouros, On the theory of hyperrings and hyperfields, Algebra i Logika, 24 (1985), 728-742.

[13] F. Marty, Sur une generalization de group, In: $8^{\text {th }}$ Congres Math. Scandinaves: Stockholm, (1934), 45-49.

[14] J. Mittas, Hypergroupes Canoniques, Mathematica Balkanica, 2 (1972), 165-179.

[15] A. Nakassis, Expository and Survey Article Recent Result in hyperring and Hyperfield Theory, Internet. J. Math and Math. Sci., 11 (2) (1988), 209-220.

[16] W. K. Nicholson, Lifting idempotents and exchange rings, Trans. Amer. Math. Soc., 229 (1977), $278-279$.

[17] W. K. Nicholson and Y. Zhou, Clean general rings, J. Algebra, 291 (2005), No. 1, 297-311.

[18] T. Vougiouklis, Hyperstructures and their representations, Hardonic, press, Inc (1994).

\section{Yahya Talebi}

Department of Mathematics, Faculty of Mathematical Sciences, University of Mazandaran, Babolsar, Iran.

talebi@umz.ac.ir

\section{Marziyeh Farzinejad}

Department of Mathematics, Faculty of Mathematical Sciences, University of Mazandaran, Babolsar, Iran

m_farzi66@yahoo.com 